Kenntnis ihrer elastomechanischen Eigenschaften und ihres Verhaltens unter Dauerbelastung. Prof. Plath (Karlsruhe) fand bei Dauerbiegeversuchen an Furnier-, Tischler- und Spanplatten im Wechselklima keine besorgniserregenden Kriecheigenschaften, sie werden jedoch durch Feuchtigkeitseinflüsse verstärkt; Holzwerkstoffe in Tragwerken müssen deshalb durch einen wasser- und wasserdampfdichten Anstrich geschützt werden. Versuche an großen Biegeträgern mit Stegen aus Spanund Hartfaserplatten bestätigten deren gute Brauchbarkeit für diesen Zweck (Prof. Cizek, Prag). Dipl.-Ing. Cziesielski (Berlin) stellte auf Grund seiner Untersuchungen Bemessungsregeln für geleimte Träger mit Stegen aus Furnierplatten und Gurten aus Vollholz bzw. aus verleimten Brettlamellen auf. Die Bemessung eines solchen Trägers muß einen allgemeinen Spannungsnachweis, einen Durchbiegungsnachweis sowie den Nachweis der Beul- und Kippsicherheit umfassen. Ein Vortrag von Dipl.-Arch. WürGLER (Zürich) über „Planen und Bauen mit Holzwerkstoffen" gab abschließend nochmals einen allgemeinen Überblick über die vielseitigen Verwendungsmöglichkeiten von Holzwerkstoffen im Bauwesen. Verschiedene Exkursionen boten Gelegenheit zur Besichtigung von interessanten Beispielen zu den besprochenen Themen und rundeten das reiche Tagungsprogramm ab.

\title{
Forstwissenschaftliche Hochschultagung 1966
}

Die Staatswirtschaftliche Fakultät der Ludwig-Maximilians-Universität und die Forstliche Forschungsanstalt München veranstalten in der Zeit vom 26. bis 29. Oktober die Forstwissenschaftliche Hochschultagung.

\section{BUCHBESPRECHUNGEN}

Forstliche Rechtslehre. Von Professor Dr. oec. publ. Dr. jur. Dr. h. c. Kurt Mantel. Verlag J. Neumann-Neudamm, Melsungen 1964. Format $17 \times 24 \mathrm{~cm}, \mathrm{XVI}, 416 \mathrm{~S}$., Kunstleinen 42,- DM.

Die vorliegende Forstliche Rechtslehre war nach dem Vorwort des Verfassers ursprünglich als eine Lieferung des Neudammer Forstlichen Lehrbuches gedacht. Der große Umfang, der sich bei einer Darstellung der forstlichen Rechtsverhältnisse ergab, und der an den Verfasser herangetragene Wunsch nach einer selbständigen Handausgabe des Forstrechts gaben dann Veranlassung zur Veröffentlichung in Buchform. Die in den letzten Jahren nach und nach erlassenen Vorschriften hinsichtlich Grundstücksrecht, Verkehrsrecht, Landesplanung, Wasserrecht, Bauordnung, Naturschutzrecht, Flurbereinigung, Arbeits- und Versicherungsrecht usw. hatten eine häufige Umarbeitung der Materie erfordert und somit die Herausgabe des Werkes leider immer wieder verzögert.

Das öffentliche Recht (ohne Strafrecht), soweit es die Forstwirtschaft berührt, bildet den Ausgangspunkt der Forstlichen Rechtslehre. In jeweils eigenen Abschnitten behandelt MaNTEL das Verwaltungsrecht (Staatliche Grundlagen, Staatsverwaltung, Selbstverwaltung), das öffentliche Eigentumsrecht (öffentliches Eigentum und Gemeinnutzung, das Privateigentum in der öffentlichen Ordnung) und das Sozialrecht (die Ordnung der Arbeit, Sozialhilfe und Sozialversicherung, das Recht des öffentlichen Dienstes). Die Forstgesetzgebung blieb der Behandlung in einem forstpolitischen Werk vorbehalten. Das Forstzivilrecbt beinhaltet das Personen- und Verbandsrecht, das Sachenrecht, das Eigentum und andere dingliche Rechte, wie z. B. Erbbaurecht, Nachbarrecht, Dienstbarkeiten, Reallasten, Nießbrauch, Grundpfandrechte usw. Die für die Forstwirtschaft wichtigen Rechtsgeschäfte (Kauf, Miete, Pacht, Dienst- 